\title{
The Role of Energy Balance in Cancer Prevention
}

\author{
Marian L. Neuhouser • Martine M. Perrigue
}

Published online: 17 January 2012

(C) Springer Science+Business Media, LLC 2012

\begin{abstract}
Nearly two thirds of American adults are overweight or obese, placing them at increased risk of obesityrelated chronic diseases, including cancer. Although it has been known for quite some time that obesity increases initial risk of breast, colon, and endometrial cancer, new data implicate obesity as a risk factor for ovarian and thyroid cancers. In addition, recent evidence shows that obesity is associated with poor survival from most cancers. Obesity influences several biological pathways, including those related to glucose homeostasis, insulin-like growth factor signaling, and inflammation. Dysregulation of these pathways in obesity may provide the biological link between obesity and carcinogenesis.
\end{abstract}

Keywords Energy balance - Overweight - Obesity - Weight loss - Cancer - Cancer risk - Cancer prevention - Breast cancer- Gynecological cancer. Thyroid cancer - Colorectal cancer - Energy restriction - Caloric restriction

\section{Introduction}

More than one half of the adult population of the United States is overweight or obese [1,2]. Of particular concern is the alarming trend in the prevalence of obesity over the past 30 years [3]. US surveillance data collected as part of the National Health and Nutrition Examination Surveys (NHANES) showed that during the period of NHANES II

\footnotetext{
M. L. Neuhouser $(\triangle) \cdot$ M. M. Perrigue

Fred Hutchinson Cancer Research Center,

1100 Fairview Avenue North, M4-B402,

Seattle, WA 98109-1024, USA

e-mail:mneuhous@fhcrc.org

M. M. Perrigue

e-mail: mperrigu@fhcrc.org
}

from 1976 to 1980, approximately 15\% of US adults were obese (defined as body mass index $[\mathrm{BMI}] \geq 30.0 \mathrm{~kg} / \mathrm{m}^{2}$ ), which rose to $23.3 \%$ during NHANES III (1988-1994). The latest NHANES estimates for data collected in 2007 and 2008 found that $34.4 \%$ of adults aged 20 to 74 years were overweight, $33.9 \%$ were obese, and $5.7 \%$ were extremely obese (BMI $\geq 40.0)$ [ $4 \bullet \bullet$. This obesity epidemic represents a major public health problem for the United States, and research to identify effective interventions for prevention and treatment of obesity are urgently needed to reverse these trends.

One of the serious consequences of the obesity epidemic is the increasing evidence that obesity is associated with increased risk of cancers of the colon, kidney, esophagus, endometrium, and postmenopausal breast. In 2002, the International Agency for Research on Cancer (IARC) issued a report stating that obesity may be responsible for as many as one quarter of cases of breast cancer and one third of cases of colon cancer [5]. The 2007 World Cancer Research Fund report on Food, Nutrition, Physical Activity, and the Prevention of Cancer indicated that there was convincing evidence that obesity and body fatness increased the risk of cancers of the esophagus, pancreas, colorectum, postmenopausal breast, endometrium, and kidney; probable evidence to support a positive association of obesity with gallbladder cancer; and limited evidence for liver and lung cancer [6]. Obesity is also well-known to increase the risk of cancer mortality. Calle and colleagues [7] reported that compared with men and women of healthy weight, for those who were very obese (BMI $>40.0 \mathrm{~kg} / \mathrm{m}^{2}$ ), the relative risk (RR) of death from cancer was 1.52 for women and 1.62 for men. The American Cancer Society, the IARC, the National Center for Health Statistics, and many other medical and consumer organizations have declared that a high priority should be placed on research in energetics and cancer to prevent a societal burden of health care costs and economic 
consequences of unprecedented proportion. Herein we review the pertinent literature on energy balance and cancer prevention published in 2010 and 2011, provide supporting biological mechanisms that may explain the energy balancecancer association, and offer provocative emerging hypotheses that may be relevant for public health programs for prevention and control.

\section{Breast Cancer}

Energy balance has been studied extensively in relation to breast cancer incidence and breast cancer survival. The 2007 World Cancer Research Fund report noted probable evidence that body fatness increased risk of premenopausal breast cancer and convincing evidence for a positive association between body fatness and postmenopausal breast cancer [6]. Data are less clear for premenopausal breast cancer. More recently, Renehan et al. [8••] published a report using data from 30 European countries examining obesity and cancer incidence and reported that nearly $5 \%$ of new postmenopausal breast cancer cases were attributable to obesity.

Four population-based cohorts, two based in the United States and two based in Japan, recently reported on weight and adult weight change in relation to breast cancer risk. The American Cancer Society's Cancer Prevention Study II cohort reported no association between weight change and weight loss measured over a 10-year period and postmenopausal breast cancer risk [9]. In the Nurses' Health Study (all postmenopausal women) and the Nurses' Health Study II (all premenopausal women), neither weight loss nor weight gain since age 18 years was associated with breast cancer risk [10]. Kawai et al. [11] assessed the relationship between current BMI, BMI change since age 20, and the risk of breast cancer in 10,106 postmenopausal Japanese women 40 to 64 years of age. Compared with women with current BMI less than $20.5 \mathrm{~kg} / \mathrm{m}^{2}$, those with a BMI of 25.9 or greater had a 2.5-fold increased breast cancer risk [11]. However, higher versus lower BMI at age 20 was inversely associated with breast cancer risk, but the upper quartile BMI cut point was $23.8 \mathrm{~kg} / \mathrm{m}^{2}$, suggesting that few in the cohort were obese at age 20. Regarding weight change, women who lost 5 or more $\mathrm{kg}$ since age 20 had a $65 \%$ lower risk of breast cancer compared with those with no or minimal weight change. However, women who gained more than $12 \mathrm{~kg}$ had a 1.5-fold increased risk of breast cancer [11]. The Japan Public Health Center cohort also reported on current BMI and change in BMI since age 20 in relation to breast cancer risk. The investigators reported that that lower BMI at age 20 (18.5-19.9 vs $20.0-23.9 \mathrm{~kg} / \mathrm{m}^{2}$ ) was associated with increased breast cancer risk (hazard ratio [HR], 1.45 [95\% CI, 1.14-1.83]), with a slightly stronger association for premenopausal breast cancer (HR, 1.57 [95\% CI, 1.142.18]) [12]. Recent or current BMI was only associated with postmenopausal breast cancer (RR, 1.31 [95\% CI, 1.071.59] for each $5-\mathrm{kg} / \mathrm{m}^{2}$ increase in BMI). Any change in BMI from age 20 years to current age was not associated with premenopausal breast cancer, and modest gains (increase of $2.5-4.9 \mathrm{~kg} / \mathrm{m}^{2}$ ) were not associated with postmenopausal breast cancer. However, large changes in BMI $\left(>5 \mathrm{~kg} / \mathrm{m}^{2}\right)$ were associated with a 1.7 -fold increased risk in postmenopausal breast cancer [12]. When analyses were stratified by tumor hormone receptor status, there was a suggestion that BMI at age 20 was inversely associated with estrogen receptor (ER)-negative/progesterone receptor (PR)negative tumors (RR, 0.49 [95\% CI, $0.27-0.88]$ per $5 \mathrm{~kg} / \mathrm{m}^{2}$ ), while risk was doubled for ER-positive/PR-positive tumors for change in weight since age 20 among postmenopausal women [12]. These results suggest that weight gain over the adult years, but not absolute weight at any one point in time, may be an important risk factor for postmenopausal breast cancer but was associated with a $32 \%$ increased risk of each among postmenopausal women [12]. Finally, a report from the Breast Cancer Association Consortium Studies reported that BMI may have differential associations with breast cancer risk based on tumor characteristics [13]. Among the 34 studies participating in the consortium, obese women younger than 50 years of age had a greater risk of ERnegative, ER-negative/PR-negative, or ER-negative/PRnegative/HER2-negative tumors compared with the risk of ER-positive tumors [13]. For women older than 50 years of age, there was a lower risk of PR-negative and ER-positive/ PR-negative tumors among the obese and no associations with any of the other tumor phenotypes [13]. Clearly, a great deal remains to be learned about the association of obesity with breast tumor characteristics, particularly with regard to obesity with other phenotypic characteristics, such as luminal $\mathrm{A}$ and $\mathrm{B}$ or basal-like tumors, for which a paucity of data currently exists.

Obesity is increasingly recognized as an important risk factor for breast cancer survival [14]. Ewertz and colleagues [15] identified 53,816 women treated for early-stage breast cancer from the Danish Breast Cancer Cooperate Group and examined associations of obesity with risk of breast cancer recurrence, breast cancer-specific mortality, and total mortality. Women who had a BMI of $30.0 \mathrm{~kg} / \mathrm{m}^{2}$ or greater had a $46 \%$ increased risk of developing distant metastases after 10 years and a $38 \%$ and $31 \%$ increased risk of breast cancer-specific death or any death, respectively, compared with women with a BMI less than $25.0 \mathrm{~kg} / \mathrm{m}^{2}$. Furthermore, the authors reported that chemotherapy and other adjuvant treatments may be less effective after 10 years or more in obese women [15]. In the California Teachers Study cohort, compared with healthy weight participants, those who were underweight or obese had an 
increased risk of total mortality (RR, 1.33 and 1.27, respectively). When results were stratified by never versus ever smoking and by never versus ever use of postmenopausal hormone therapy, results were attenuated but remained significant only for total mortality [16]. Finally, in the Shanghai Breast Cancer Survival study, women who were obese at diagnosis had a 1.5 -fold increased risk of total mortality and a 1.4-fold increased risk of breast cancer recurrence or disease-specific mortality compared with women who were not overweight or obese [17]. In addition, weight change after diagnosis was associated with outcomes in which women who gained at least $5 \mathrm{~kg}$ or lost at least $1 \mathrm{~kg}$ after diagnosis had poor outcomes compared with women who remained weight stable after diagnosis [17]. It is important to further clarify the role of obesity in breast cancer survival.

Obesity disrupts multiple metabolic pathways, including those related to inflammation, insulin resistance, insulinlike growth factor pathway signaling, and subsequent downstream molecules (including mammalian target of rapamycin [mTOR]) [18], 19, 20••, 21, 22]. Clinical trials and observational studies are under way to help us better understand which of these obesity-related pathways might respond best to lifestyle modifications or pharmacologic interventions. For example, a clinical trial is under way to test whether metformin can effectively improve survival in breast cancer patients [23]. Energy restriction is one lifestyle modification approach under investigation. Although energy restriction has been effective consistently for reducing incidence and severity of mammary cancer in preclinical animal models, there is a paucity of data showing the impact of long-term energy restriction on breast cancer risk, and studies on short-term, transient energy restriction and breast cancer incidence and survival in humans are inconclusive [24].

Summary Statement for Energy Balance and Breast Cancer

There is consistent evidence for a positive association between obesity and risk of breast cancer and poor prognosis in postmenopausal women; data are less clear regarding risk and prognosis for premenopausal breast cancer. Long-term energy balance, considered in terms of life span weight gain or loss, may be an additional important predictor risk for breast cancer and may provide additional clues to the mechanisms driving cancer development. As such, measurement of life span weight status, including weight change, should be incorporated into studies of breast cancer risk and prognosis whenever possible. Further research is still needed to help us better understand energy balance in relation to premenopausal breast cancer and whether obesity differentially impacts various tumor phenotypes, such as triple-negative tumors, luminal A and $\mathrm{B}$ tumors, and basal-like tumors.

\section{Colorectal Cancer}

The 2007 World Cancer Research Fund Report noted that evidence is convincing for a positive association between body fatness and abdominal fatness and colorectal cancer risk [6]. Since this 2007 report, Renehan and colleagues [8••] reported from a large European study that $10.9 \%$ of all colorectal cancer cases in men and $2.6 \%$ of colorectal cancer cases in women were attributable to BMI. Each BMI increase of $5 \mathrm{~kg} / \mathrm{m}^{2}$ was associated with a colon cancer RR of $1.21(95 \%$ CI, 1.18-1.23) in men and 1.04 (95\% CI, 1.00-1.10) in women. The relationship of obesity to rectal cancer was less clear, partially due to there being fewer cases [8••]. More recently, in the European Prospective Investigation into Cancer and Nutrition (EPIC) study, investigators reported that abdominal obesity, defined as high waist circumference, was associated with an approximately twofold increase in colon cancer risk for men, but not women [25]. However, there were no associations of abdominal obesity with rectal cancer risk [25]. Similarly, participants in the Singapore Chinese Health study who were overweight or obese at study entry had an RR for colon and colorectal cancer of 1.48 and 1.25 , respectively, but there was no association of BMI with rectal cancer [26]. The Iowa Women's Health study reported that several measures of energy balance (weight, BMI, hip circumference, waist circumference, and waist-hip ratio) were associated with colorectal cancer risk; the HRs ranged between 1.4 and 1.6 for high versus low for each of these measures of obesity [27]. Associations were slightly stronger for distal versus proximal tumors. Obesity measures, including change in weight at specific ages, were also evaluated. BMI at ages 40 and 50 was suggestively associated with increased colorectal cancer risk, but BMI at ages 18 and 30 was only weakly or not at all associated with colorectal cancer risk. Similarly, there were no differences in risk estimates by menopausal status [27]. These results are similar to those from the Melbourne Collaborative study, which reported that neither BMI nor weight at age 18 was associated with colon cancer risk and that adult weight gain in men, but not women, was associated with an HR of 1.11 for each 5-kg increase in weight during adulthood [28].

Energy balance also has been explored as a prognostic indicator for colon cancer survival. Studies investigating the relationship between obesity and risk for colon cancer recurrence and mortality have yielded mixed results. Using data from 7 randomized controlled trials of adjuvant therapy for colorectal cancer, researchers recently examined associations among BMI, gender, DNA mismatch repair status, response to adjuvant therapy, and colon cancer prognosis in 4,381 patients with curatively resected stage II and III colon cancers [29•]. In men, a BMI of $35 \mathrm{~kg} / \mathrm{m}^{2}$ or greater was associated with lower overall survival compared with a BMI of 20 to $24.9 \mathrm{~kg} / \mathrm{m}^{2}$ (HR, 1.35 [95\% CI, $1.02-$ 
1.79]). In women, a BMI of 30.0 to $34.9 \mathrm{~kg} / \mathrm{m}^{2}$ was associated with lower overall survival compared with a BMI of 20.0 to $24.9 \mathrm{~kg} / \mathrm{m}^{2}$ (HR, 1.24 [95\% CI, 1.01-1.53]). The authors concluded that obesity is a strong prognostic variable for colon cancer survival, but that the strength of the association may differ between men and women [29•]. Despite the weaker observed associations in females for obesity and colorectal cancer survival in the aforementioned study, the Iowa Women's Health study recently reported that both baseline weight and BMI were strong and independent predictors of death from distant stage colorectal cancer [30].

Recent epidemiologic studies have sought to better understand the relationship between long- and short-term energy balance and risk of colorectal cancer by considering life span weight status, periods of energy restriction, and tumor characteristics. In a 2003 study using data from the Netherlands Cohort study, investigators found a weak, nonsignificant relationship between transient energy restriction during childhood and adolescence and subsequent colon cancer [31]. Using the same dataset, Hughes et al. [32] conducted a case-cohort study to determine whether energy restriction during childhood and adolescence lowers cancer risk at multiple sites. Men with the highest degree of energy restriction had a lower risk of developing colorectal cancer (RR, 0.81 [95\% CI, 0.68-0.98]) and tumors of the proximal colon (RR, 0.72 [95\% CI, 0.54-0.96]) and rectum (RR, 0.71 [95\% CI, 0.53-0.96]) [32].

\section{Summary Statement for Energy Balance and Colorectal Cancer}

Evidence continues to support a positive association of obesity with colon cancer, but data are less clear with regard to rectal cancer risk. In addition, data are inconsistent with respect to gender differences in risk, some of which may be confounded by postmenopausal hormone therapy use by females. Emerging data suggest that weight gain and patterns of weight history may influence colorectal cancer risk.

\section{Gynecological Cancer}

Studies consistently demonstrate a positive association between BMI and risk of endometrial cancer, including a rating of convincing evidence from the 2007 World Cancer Research Fund Report [6, 33, 34]. A meta-analysis using data from 19 prospective cohort studies indicated a strong positive association between increased BMI and endometrial cancer risk (RR, 1.59 [95\% CI, 1.50-1.68]; $P<0.0001$ ) [8••]. In addition, weight gain during young adulthood is associated with increased risk of endometrial cancer [6, 35, 36]. More recently, Park et al. [37] investigated the relationship between body size and risk of endometrial cancer and whether associations varied by race/ethnicity among 50,376 women enrolled in the Multiethnic Cohort study. Compared with the reference group, $(-5 \% \leq \mathrm{BMI}$ change $\leq+5 \%)$, women who gained $35 \%$ or more in BMI from age 21 to the time of study recruitment had increased risk of endometrial cancer (RR, 4.12 [95\% CI, 2.60-6.30]). Compared with women who maintained a stable BMI $(-0.25 \%$ to $<+0.25 \%)$, women who gained an average of $1 \%$ or more per year had a more than 3.21-fold increased risk (95\% CI, 2.37-4.33) of endometrial cancer. Among all racial/ethnic groups in this diverse cohort, there was a positive association among weight, BMI, and BMI change and risk of endometrial cancer. In subgroup analyses by race/ethnicity, for Japanese American women, a smaller BMI gain ( $\geq 5 \%$ from age 21 to time of study recruitment) was associated with increased risk, whereas other race/ethnicities needed a larger BMI increase to influence risk of endometrial cancer [37].

Few studies have examined whether energy balance influences ovarian cancer risk. However, three recent cohort studies have provided evidence linking energy balance with ovarian cancer risk. The Melbourne Collaborative study, the California Teachers study, and EPIC all reported evidence to support a positive association of obesity with ovarian cancer, but the types of obesity measures conferring risk varied across the cohorts. In the Melbourne cohort, BMI (per $5-\mathrm{kg} / \mathrm{m}^{2}$ increase) and fat mass (per 10-kg increase) were both associated with modest (RR, 1.2 and 1.3, respectively) but statistically significant results [38]. EPIC reported that BMI was associated with a modest HR of 1.38 in postmenopausal women, but other measures of adiposity, such as weight gain, waist circumference, and waist-hip ratio, were not associated with risk in premenopausal or postmenopausal women [39]. In contrast, the Teachers cohort reported that adult weight change and waist circumference, but not BMI, independently increased ovarian cancer risk nearly twofold $[40 \bullet \cdot]$. One of the limitations of these studies is the relatively small number of cases, as ovarian cancer is a rare cancer. Ovarian cancer studies are further confounded by the fact that clinical symptoms include weight gain and increasing abdominal girth, which often precede the diagnosis by as much as a few years. Collaborative studies using data from multiple cohorts are needed to help us better understand the association between energy balance and ovarian cancer risk.

Summary Statement for Energy Balance and Gynecological Cancer

Strong and relatively consistent evidence from prior studies supports an association of positive energy balance with risk of endometrial cancer. Percentage change in BMI, indicative of long-term energy balance, is an important risk factor for endometrial cancer, and new evidence suggests that this association may be influenced by race/ethnicity, in which the 
"necessary dose of obesity" may differ by race/ethnicity. Few studies have examined energy balance and ovarian cancer. Although results are suggestive that obesity is associated with ovarian cancer risk, data are insufficient at this time to specify which obesity risk factors confer the strongest risk.

\section{Thyroid Cancer}

Incidence rates of thyroid cancer have increased dramatically over the past three decades in the United States [41]. This may be attributable - at least in part - to the concurrent rise in overweight and obesity [42••]. BMI was positively associated with thyroid cancer in men (RR, 1.33 [95\% CI, 1.04-1.70]; $P<0.02$ ) and women (RR, 1.14 [95\% CI, 1.06-1.23]; $P<0.0001)$ in an analysis of prospective cohort studies, although limited data were available $[8 \cdot \bullet]$. The relationship among BMI, physical activity, and thyroid cancer was recently examined in a prospective cohort study of 484,326 participants from the National Institutes of Health-American Association of Retired Persons (NIH-AARP) Diet and Health study [42*0]. Compared with healthy weight participants, overweight and obese individuals had a modest but significant increased risk of thyroid cancer (RR, $1.27[95 \% \mathrm{CI}$, 0.99-1.63] for overweight and 1.39 [95\% CI, 1.05-1.85] for obese; $P$ for trend $<0.007)$. Findings appeared to be somewhat stronger for papillary thyroid tumors versus other tumors, but with fewer cases in the subgroup analyses, the power to detect differences was diminished [42••]. In a subsequent report in the NIH-AARP cohort, waist circumference was associated with an RR of thyroid cancer of 1.8 for men and 1.5 for women, and high BMI plus high waist circumference doubled the risk in men and women. However, adult weight gain was more strongly associated with thyroid cancer risk in men than in women [43••]. A cohort study of women in France indicated that adult weight gain (assessed as change in body shape) yielded a nearly twofold increased risk of thyroid cancer [44]. Finally, a recent pooled analysis from five US-based cohorts assessed the association of weight with thyroid cancer [45]. The pooled HRs were modest (per $5-\mathrm{kg} / \mathrm{m}^{2}$ : HR, 1.16 [95\% CI, 1.08-1.24] for women and 1.21 [95\% CI, 0.97-1.49] for men) [45]. There were also modest but significant data showing that BMI in early-adulthood is an independent predictor of thyroid cancer (per 5-kg/m²: HR, 1.18 [95\% CI, 1.03-1.35]) [45].

Summary Statement for Energy Balance and Thyroid Cancer

New data are emerging to support an association of energy balance with thyroid cancer risk. In the near future, it would be beneficial for cohorts to conduct additional pooled analyses, as the number of cases from each cohort may provide sufficient power to detect associations. Because the incidence of thyroid cancer is increasing, these types of collaborative efforts should be research priorities.

\section{Emerging Hypotheses Related to Energy Balance and Cancer}

Energy Balance Throughout the Life Span: What is the Most Critical Time Period for Cancer Prevention?

The American Cancer Society's Guidelines on Nutrition for Cancer Prevention include recommendations to maintain a healthy weight throughout life, consume a healthy diet with at least five daily servings of fruits and vegetables, consume whole grains instead of processed grains, limit consumption of processed and red meats, and to use alcohol in moderation [46]. These guidelines are similar to the United States Dietary Guidelines, which are dietary recommendations for general health promotion and disease prevention for all Americans. Despite the rather general recommendations to "maintain a healthy weight" for cancer prevention, interesting data have emerged recently that suggest that the pattern and timing of adult weight gain may be a more important predictor of cancer than simply absolute weight gain or BMI at a single point in time.

BMI measured at a single point in time historically has been used as the primary energy balance exposure assessment in observational studies. More recently, data are being collected to assess weight and weight patterns throughout the life span. Although this approach may be subject to some degree of measurement error due to participant reliance on memory of past weight, these data clearly will help researchers better understand the role of energy balance throughout the life span in relation to risk, prognosis, and mechanisms underlying cancer development. For example, in recent studies in which adult weight change was measured, weight gain was positively associated with risk of breast, colorectal, ovarian, endometrial, and thyroid cancers. Summary data presented in this report suggest that long-term energy balance status may play a critical role with regard to determining cancer risk. Clinicians and leaders of local and national public health programs can use this information to provide yet another important reason to maintain a healthy weight.

\section{Does Energy Restriction Influence Cancer Risk?}

Diet is a modifiable risk factor for cancer incidence and cancer survival. Data from observational studies in humans suggest that restriction of energy intake may be an effective strategy for cancer prevention [47, 48•]. Investigators have used a variety of animal models to demonstrate beneficial effects of energy restriction, including increased life span 
and lower cancer rates or less severe disease, in preclinical models $[47,48] \cdot$. Given this evidence, some of the most intriguing emerging strategies for lowering cancer risk involve energy restriction through reduced caloric intake on a chronic or intermittent basis. Several recent investigations provide insight into the effects of energy restriction on cancer risk and possible underlying mechanisms.

Limited evidence supports a relationship between restricted energy intake and prevention of cancer in humans, due in large part to the ethical challenges inherent in such study designs. Historical events such as war, famine, and periods of economic depression have provided opportunities to investigate the effects of short-term energy restriction on risk of a variety of cancer types. Risk of breast cancer was significantly higher in women exposed to severe, transient energy restriction compared with those not exposed during the 1944 to 1945 Dutch famine [31]. Colorectal cancer risk was lower in males exposed to the highest level of transient energy restriction during periods of severe famine, economic depression, and wartime [32]. As noted in a recent review, interpretation of these results can be difficult due to confounding factors associated with conditions of extreme stress [48•]. Although data are sparse, studies indicate that transient energy restriction during critical periods of development such as childhood and adolescence can impact risk of cancer later in life. Cancer risk may differ according to gender, age, and pubertal status at time of exposure; severity and duration of energy restriction; and cancer location and subtype. Proxy measures and self-reported data often must be used to estimate diet quality, degree of energy restriction, body weight, and other important information [32]. Further research needs to be conducted to determine the impact of short-term, transient energy restriction on cancer risk and to elucidate any potential mechanisms.

In light of the extensive evidence linking excess adiposity with carcinogenesis and current worldwide health trends, the urgent need for strategies combining prevention of obesity and reducing cancer risk is quite clear. In recent years, chronic caloric restriction (CR) without malnutrition has gained considerable attention as a means to increase longevity and decrease risk of cancer [49, 50]. The CALERIE study (Comprehensive Assessment of the Long-term Effect of Reducing Intake of Energy), was a large, randomized controlled trial of CR conducted in humans. In phase 1 of the study (6 months), 48 men and women were randomly assigned to 1 of 4 groups: CR ( $25 \% \mathrm{CR})$, CR plus exercise (25\% energy deficit achieved by $12.5 \% \mathrm{CR}$ and $12.5 \%$ increased energy expenditure), low-calorie diet (consumption of $890 \mathrm{kcal} / \mathrm{d}$ until $15 \%$ weight loss, followed by weight maintenance diet), or healthy diet control (weight-maintaining diet based on American Heart Association step 1 diet) [50]. Results from studies using data from CALERIE indicate that $\mathrm{CR}$ decreases whole body oxygen consumption and DNA damage [51], reduces insulin resistance [52], and modulates markers of oxidative stress in humans [53]. Redman and colleagues [54] found that CR alone did not modulate growth hormone secretory dynamics, but that significant alterations in growth hormone and insulin-like growth factor-1 profiles were induced by other regimens.

Long-term adherence to a calorically restricted diet regimen is unrealistic for many individuals. Various forms of intermittent fasting involving reduced overall caloric intake have been suggested as a more manageable alternative to long-term CR for cancer risk reduction [55]. Harvie and colleagues [56] recently compared the effects of a $25 \%$ total energy restriction as either intermittent energy restriction (IER; $2 \mathrm{~d} / \mathrm{wk} 75 \%$ energy restriction, $5 \mathrm{~d} / \mathrm{wk}$ ad libitum consumption) or continuous energy restriction (CER; $7 \mathrm{~d} / \mathrm{wk}$ $25 \%$ energy restriction) for a period of 6 months. Participants were 107 overweight and obese premenopausal women. Both treatment groups showed significant weight loss and reductions in C-reactive protein and markers of risk of breast cancer [56]. Results were comparable between the IER and CER groups, supporting use of IER as an alternative to CER for weight loss and disease prevention [56].

Weight loss via energy restriction may reduce the risk of cancer via modification of various regulatory pathways. Previous investigators have reviewed the mechanisms involved in cancer prevention through CR [47, 49, 50]. Perhaps the most well-studied of these is the circulating insulin-like growth factor system. In addition to energy content, the macronutrient composition of the diet may direct changes in the insulin-like growth factor system. In a recent study, Belobrajdic and colleagues [57] provided overweight and obese male participants an energy-restricted diet $(7,000 \mathrm{~kJ} / \mathrm{d})$ of high-protein or high-carbohydrate foods. Weight loss and modulation of the insulin-like growth factor system were similar in both groups, indicating that energy restriction and the resulting weight loss, rather than diet composition, directed the observed changes [57].

Although the evidence suggests that CR may influence several of the key biological pathways involved in carcinogenesis, much remains to be learned before CR can be offered as an effective cancer prevention strategy in humans. Investigators have yet to determine whether energy restriction at certain life stages is associated with greater risk than benefit, or whether energy restriction may increase the risk of some cancers and decrease the risk of others. Questions remain regarding an optimal duration and degree of energy restriction and whether energy restriction should be on a chronic or intermittent basis. Additionally, diet composition and physical activity deserve further exploration, as they may provide additional benefits to those following energy-restricted diet regimens. 


\section{Conclusions}

Evidence continues to accumulate demonstrating strong associations between energy balance and cancer risk. Questions remain as to whether life span weight gain, recent weight change, or weight change over the adult period is the most important risk factor. Nonetheless, clinicians and public health officials should continue to advocate for programs to prevent and treat obesity.

Disclosure No potential conflicts of interest relevant to this article were reported.

\section{References}

Papers of particular interest, published recently, have been highlighted as:

- Of importance

-• Of major importance

1. Flegal KM, Carroll M, Ogden CL, Johnson C. Prevalence and trends in obesity among US adults. JAMA. 2002;288:1723-7.

2. Ogden CL, Carroll MD, Curtin LR, et al. Prevalence of overweight and obesity in the United States, 1999-2004. JAMA. 2006;295:1549-55.

3. Flegal KM, Carroll MD, Kuczmarski RJ, Johnson CL. Overweight and obesity in the United States: prevalence and trends, 1960-1994. Int J Obesity. 1998;22:39-47.

4. •- Flegal KM, Carroll MD, Ogden CL, Curtin LR: Prevalence and trends in obesity among US adults, 1999-2008. JAMA 2010;303:235-41. This article presents the most current trends and data on obesity in the United States.

5. IARC. IARC Working Group on the Evaluation of CancerPreventive Strategies. In: IARC Press, ed. Weight Control and Physical Activity, IARC Handbook of Cancer Prevention. Lyon, France, 2002:215.

6. World Cancer Research Fund/AICR. Food, Nutrition, Physical Activity, and the Prevention of Cancer: a Global Perspective. Washington, DC: AICR; 2007.

7. Calle R, Rodriguez C, Walker-Thurmond K, Thun M. Overweight, obesity, and mortality from cancer in a prospectively studied cohort of U.S. adults. N Engl J Med. 2003;348:1625-38.

8. •• Renehan AG, Soerjomataram I, Tyson M, et al.: Incident cancer burden attributable to excess body mass index in 30 European countries. Int J Cancer 2010;126:692-702. This article presents important data on the population excess burden of cancer attributable to obesity.

9. Teras LR, Goodman MT, Patel AV, et al. Weight loss and postmenopausal breast cancer in a prospective cohort of overweight and obese US women. Cancer Causes \& Control. 2011;22:573-9.

10. Michels KB, Terry KL, Eliassen AH, et al.: Adult weight change and incidence of premenopausal breast cancer. Int J Cancer epub, 2011.

11. Kawai M, Minami Y, Kurlyama S, et al. Adiposity, adult weight change and breast cancer risk in postmenopausal Japanese women: the Miyagi Cohort Study. Br J Cancer. 2010;103:1443-7.

12. Suzuki R, Iwasaki $M$, Inouee $M$, et al. Body weight at age 20 years, subsequent weight change and breast cancer risk defined by estrogen and progesterone receptor status-The Japan Public
Health Center-based Prospective Study. Int J Cancer. 2011;129: 1214-24.

13. Yang XR, Chang-Claude J, Goode EL, et al. Associations of breast cancer risk factors with tumor subtypes: a pooled analysis from the Breast Cancer Association Consortium studies. J Natl Cancer Inst. 2011;103:250-63.

14. de Azambuja E, McCaskill-Stevens W, Francis P, et al. The effect of body mass index on overall and disease-free survival in node-positive breast cancer patients treated with docetaxel and doxorubicincontaining adjuvant chemotherapy: the experience of the BIG 0298 trial. Breast Cancer Research and Treatment. 2010;119:145-53.

15. Ewertz M, Jensen MB, Gunnarsdóttir KÁ, et al. Effect of obesity on prognosis after early-stage breast cancer. J Clin Oncol. 2011;29:25-31

16. Bessonova L, Marshall SF, Ziogas A, et al. The association of body mass index with mortality in the California Teachers Study. Int J Cancer. 2011;129:2492-501.

17. Chen $\mathrm{X}, \mathrm{Lu} \mathrm{W}$, Zheng $\mathrm{W}$, et al. Obesity and weight change in relation to breast cancer survival. Breast Cancer Res Treat. 2010;122:823-33.

18. Healy LA, Ryan AM, Carroll P, et al. Metabolic syndrome, central obesity and insulin resistance are associated with adverse pathological features in postmenopausal breast cancer. Clinical Oncology. 2010;22:281-8.

19. Kahn BB, Flier JS. Obesity and insulin resistance. Journal of Clinical Investigation. 2000;106:473-81.

20. •• Parekh N, Okada T, Lu-Yao G: Obesity, insulin resistance and cancer prognosis: implications for practice for providing care among cancer survivors. J Am Diet Assoc 2009;109:1346-53. This article provides important practical data for clinicians working with cancer survivors.

21. Shoelson SE, Herrero L, Naaz A. Obesity, inflammation, and insulin resistance. Gastroenterology. 2007;132:2169-80.

22. Bosco JLF, Antonsen S, Sørensen HT, et al. Metformin and incident breast cancer among diabetic women: a population- based case-control study in Denmark. Cancer Epidemiol Biomarkers Prev. 2011;20:101.

23. Goodwin PJ, Ligibel JA. Metformin in breast cancer: time for action. J Clin Oncol. 2009;27:3271-3.

24. Haars G, van Gils CH, Elias SG, et al. The influence of a period of caloric restriction due to the Dutch famine on breast density. Int J Cancer. 2010;126:2211-5.

25. Aleksandrova $\mathrm{K}$, Boeing $\mathrm{H}$, Jenab $\mathrm{M}$, et al.: Metabolic syndrome and risks of colon and rectal cancer: the European Prospective Investigation into Cancer and Nutrition Study. Cancer Prevention Research epub, 2011.

26. Odegaard AO, Koh WP, Yu MC, Yuan JM. Body mass index and risk of colorectal cancer in Chinese Singaporeans. Cancer. 2011;117:3841-9.

27. Oxentenko AS, Bardia A, Vierkant RA, et al. Body size and incident colorectal cancer: a prospective study of older women. Cancer Prevention Research. 2010;3:1608-20.

28. Bassett JK, Severi G, English DR, et al. Body size, weight change, and risk of colon cancer. Cancer Epidemiol Biomarkers Prev. 2010;19:2978-86.

29. - Sinicrope FA, Foster NR, Sargent DJ, et al.: Obesity is an independent prognostic variable in colon cancer survivors. Clin Cancer Res 2010;16:-93. This article features interesting data on obesity and its relationship to colorectal cancer survival.

30. Prizment AE, Flood A, Anderson KE, Folsom AR. Survival of women with colon cancer in relation to precancer anthropometric characteristics: the Iowa Women's Health Study. Cancer Epidemiol Biomarkers Prev. 2010;19:2229-37.

31. Elias SG, Peeters PHM, Grobbee DE, van Noord PAH. Breast cancer risk after caloric restriction during the 1944-45 Dutch famine. J Natl Cancer Inst. 2004;96:539-46. 
32. Hughes LAE, van den Brandt PA, Goldbohm RA, et al. Childhood and adolescent energy restriction and subsequent colorectal cancer risk: results from the Netherlands Cohort Study. Int J Epidemiol. 2010;39:1333-44.

33. Salazar-Martínez E, Lazcano-Ponce EC, Lira-Lira GG, et al. Case-control study of diabetes, obesity, physical activity and risk of endometrial cancer among Mexican women. Cancer Causes Control. 2000;11:707-11.

34. Goodman MT, Hankin JH, Wilkens LR, et al. Diet, body size, physical activity, and the risk of endometrial cancer. Cancer Res. 1998;57:5077-85.

35. Schouten LJ, Goldbohm RA, van den Brandt PA. Anthropometry, physical activity, and endometrial cancer risk: results from The Netherlands Cohort Study. J Natl Cancer Inst. 2004;96:1635-8.

36. Friedenreich C, Cust A, Lahmann PH, et al. Anthropometric factors and risk of endometrial cancer: the European prospective investigation into cancer and nutrition. Cancer Causes Control. 2007;18:399-413.

37. Park SL, Goodman MT, Zhang ZF, et al. Body size, adult BMI gain and endometrial cancer risk: the multiethnic cohort. Int $\mathrm{J}$ Cancer. 2010;126:490-9.

38. Chiohn F, Baglietto L, Krishnan K, et al. Physical activity, body size and compositions, and risk of ovarian cancer. Cancer Causes \& Control. 2010;21:2183-94.

39. Lahmann PH, Cust AE, Friedenreich CM, et al. Anthropometric measures and epithelial ovarian cancer risk in the European Prospective Investigation into Cancer and Nutrition. Int J Cancer. 2010;126:2404-15.

40. • Canchola AJ, Chang ET, Bernstein L, et al.: Body size and the risk of ovarian cancer by hormone therapy use in the California Teachers Study cohort. Cancer Causes Control 2010;21:2241-8. This article presents some of the first data to document a relationship between body size and ovarian cancer risk.

41. Jemal A, Siegel R, Xu J, Ward E: Cancer Statistics, 2010. CA Cancer J Clin 2010 Epub ahead of print.

42. • Leitzmann MF, Brenner A, Moore SC, et al.: Prospective study of body mass index, physical activity and thyroid cancer. Int J Cancer 2010;126:2947-56. Little is known about the increasing prevalance of thyroid cancers; this report suggests a link between obesity and thyroid cancer and that differences occur by thyroid cancer type.

43. •- Kitahara CM, Platz EA, Park Y, et al.: Body fat distribution, weight change during adulthood, and thyroid cancer risk in the NIH AARP diet and health study. Int J Cancer e-pub 2011. Little is known about the increasing prevalance of thyroid cancers; this report suggests a link between obesity and thyroid cancer.

44. Clavel-Chapelon F, Guillas G, Tondeur L, et al. Risk of differentitated thyroid cancer in relation to adult weight, height and body shape over life: the French E3N Cohort. Int J Cancer. 2010;126:2984-90.

45. Kitahara CM, Platz EA, Beane Freeman L, et al. Obesity and thyroid cancer risk among US men and women: a pooled analysis of 5 prospective studies. Cancer Epidemiol Biomarkers Prev. 2011;20:464-72.

46. Doyle C, Kushi LH, Byers T, et al. Nutrition and physical activity during and after cancer treatment: an American Cancer Society guide for informed choices. CA Cancer J Clin. 2006;56: 323-53.

47. Hursting SD, Lavigne JA, Berrigan D, et al. Calorie restriction, aging, and cancer prevention: mechanisms of action and applicability to humans. Ann Rev Med. 2003;54:131-52.

48. - Hursting SD, Berger NA: Energy balance, host-related factors, and cancer progression. J Clin Oncol 2010;28:4058-64. This article provides data on the biological mechanisms to explain energy imbalance and carcinogenesis.

49. Longo VD, Fontana L. Calorie restriction and cancer prevention: metabolic and molecular mechanisms. Cell. 2009;31:89-98.

50. Redman LM, Ravussin E. Caloric restriction in humans: impact on physiological, psychological, and behavioral outcomes. Antioxid Redox Signal. 2011;14:275-87.

51. Civitarese AE, Carling S, Heilbronn LK, et al. Calorie restriction increases muscle mitochondrial biogenesis in healthy humans. PLoS Medicine. 2007;4:e76.

52. Larson-Meyer DE, Heilbronn LK, Redman LM, et al. Effect of calorie restriction with or without exercise on insulin sensitivity, cell function, fat cell size, and ectopic lipid in overweight subjects. Diabetes Care. 2006;29:1337-44.

53. Meydani M, Das S, Band M, et al. The effect of caloric restriction and glycemic load on measures of oxidative stress and antioxidants in humans: results from the CALERIE Trial of human caloric restriction. The Journal of Nutrition. 2011;15:456-60.

54. Redman LM, Veldhuis JD, Rood J, et al. The effect of caloric restriction interventions on growth hormone secretion in nonobese men and women. Aging Cell. 2010;9:32-9.

55. Harvie M, Howell A. Energy balance adiposity and breast cancerenergy restriction strategies for breast cancer prevention. Obes Rev. 2006;7:33-47.

56. Harvie MN, Pegington M, Mattson MP, et al. The effects of intermittent or continuous energy restriction on weight loss and metabolic disease risk markers: a randomized trial in young overweight women. Int J Obesity. 2011;35:714-27.

57. Belobrajdic DP, Frystyk J, Jeyaratnaganthan N, et al. Moderate energy restriction-induced wieght loss affects circulating IGF levels independent of dietary composiiton. Eur J Endocr. 2010;162:1075-82. 